\title{
ESPI Solution for Defect Detection in Crystalline Photovoltaic Cells
}

\author{
Ching-Chung Yin*, Tzu-Kuei Wen \\ Department of Mechanical Engineering, National Chiao Tung University \\ Hsinchu 30010, Taiwan, ROC
}

\begin{abstract}
The yield of photovoltaic (PV) cells is often reduced by micro-defects in crystalline silicon substrates during fabrication. Common optical inspection for a thin crack in such a large silicon photovoltaic cell is extremely time-consuming and fails in efficiency. This study developed a method of using electronic speckle pattern interferometry (ESPI) for rapidly testing for cracks in an entire field of PV cells. Thermally induced flexural cell deformation was measured by optical configuration for ESPI measurement of out-of-plane deformations. Experimental results indicate that the speckle patterns correlating with thermal deformation of cell enable simultaneous estimation of crack size and location in both single- and poly-crystalline PV cells. This nondestructive detection method has potential applications in PV cell sorting.
\end{abstract}

Keywords: Defect detection, photovoltaic cell, electronic speckle pattern interferometry, thermal deformation

\section{INTRODUCTION}

Photovoltaic technology has progressed rapidly in recent years. Manufacturers of PV cells tend to focus on electrical performance rather than on mechanical properties. However, mechanical defects are commonly found in brittle, crystalline silicon photovoltaic cells, which comprise over $80-90 \%$ of global market. Solar cells are usually installed outdoors in sunny areas and exposed to a huge temperature difference between day and night. Cracking is a major cause of catastrophic damage to cell modules ${ }^{[1]}$. Identifying solar cell integrity before module fabrication is essential for minimizing manufacturing cost. It results in the growing need for an effective nondestructive method which possesses fast and precise on-line fault detection capability for photovoltaic industry.

Various techniques and approaches have been used or developed for detecting defects in silicon substrates and PV cells. Electroluminescence ${ }^{[2]}$ (EL) and photoluminescence ${ }^{[3]}$ (PL) imaging are both used to characterize silicon wafers and to observe damages in solar cells. Both use charge coupled device (CCD) cameras to record the luminescence signals. These imaging methods were originally developed to test electrical performance and to identify ion-implanted defects. Although they can be used to visualize fractures, they do not clearly distinguish between very small surface scratches and cracks. Another potential imaging method for spatially resolved solar cell characterization is the illuminated lock-in thermography ${ }^{[4]}$ (ILIT), in which heat radiation from a sample is imaged by an infrared camera. This approach provides high quality imaging of cracks and damage in the sample. However, it is time-consumed for high lock-in integration which is needed to improve the signal-to-noise ratio. Resonance ultrasonic vibrations ${ }^{[5]}$ (RUV) can identify solar wafer quality according to the changes in resonant frequency and bandwidth of a sample, but it can not indicate the location of cracks or damages. The current methods have their individual advantages in inspection of solar cells and silicon wafers. No single method has been proven to be rapid and precise for solar cell damage inspection.

The electronic speckle pattern interfermetory ${ }^{[6-7]}$ (ESPI) can identify deformation on the specimen surface in accordance with its fringe pattern, which is affected by the boundary condition and original deformation of the specimen. This work presents an optical crack detection method based on ESPI method to rapidly inspect photovoltaic cells. The interference fringes are associated with contours of the heat-induced bending deflection on the inspected solar cell. The out-of-plane displacement is discontinuous across the crack surface and there remain broken tangential slopes on the interference fringes. The discontinuity of speckle fringe can be used to characterize appearance of defects and to estimate crack size in PV cells. In order to accurately predict thermal deformation and temperature rise required in heating cycles, the PV cells subject to various boundary conditions were numerically investigated using finite element analysis (FEA).

* Corresponding author: ccyin@mail.nctu.edu.tw; phone +886-3-5718965; fax +886-3-5720634

Seventh International Symposium on Precision Engineering Measurements and Instrumentation, edited by Kuang-Chao Fan, Rong-Sheng Lu, Man Song, Proc. of SPIE Vol. 8321

$832139 \cdot$ (c) 2011 SPIE · CCC code: /11/\$18 · doi: 10.1117/12.905261 


\section{EXPERIMENTAL}

Commercially available crystalline silicon photovoltaic cells $(156 \times 156 \times 0.2 \mathrm{~mm})$ were used for test specimens. The front surface of the specimen was coated with an antireflection film of thickness about $15 \mu \mathrm{m}$. A metallic ink was screenprinted on it to form the electrode pattern. The rear surface was printed with aluminum paste and two silver strips $(156 \times 4 \times 0.01 \mathrm{~mm}$ each). Wafer bow can be reduced if a mismatch of the coefficients of thermal expansion (CTE) between silicon wafer and backing aluminum paste is avoided. In spite of this concern, heat-induced flexure is caused by a difference of CTE between screen-printed electrodes and cell. In this experiment, the heating temperature of cells ranged from room temperature to 50 degrees Celsius. Thermal deformation is linearly proportional to temperature rise.

Two cracked specimens were considered as examples. There was a crack extended from an edge of the cell in the first specimen. An interior crack appeared within the second specimen. The cracks were artificially induced by pressing a small pyramid-shaped diamond indenter against the specimens using a Matsuzawa digital Rockwell hardness tester. Figure 1 shows an optical micrograph of cross section of a surface crack in a specimen. The bulk aluminum layer on the rear side of a solar cell comprises the back surface field (BSF) layer, $\mathrm{Al} / \mathrm{Si}$ eutectic layer, and porous bismuth-silicate glass matrix filled with Al-Si spherical particles surrounded by a thin aluminum oxide layer. The eutectic layer has a relatively high fracture toughness. A local crack branching or deflection could be found while the surface crack growth reaches the interface between silicon and the bulk particulate aluminum layer. The experimental results were further verified by EL imaging method using a tester, MT-EL-H1708M developed by Scientek Corp. The EL tests were operated at $1 \mathrm{~A}$ current and had a resolution of $1392 \times 1040$ pixels.

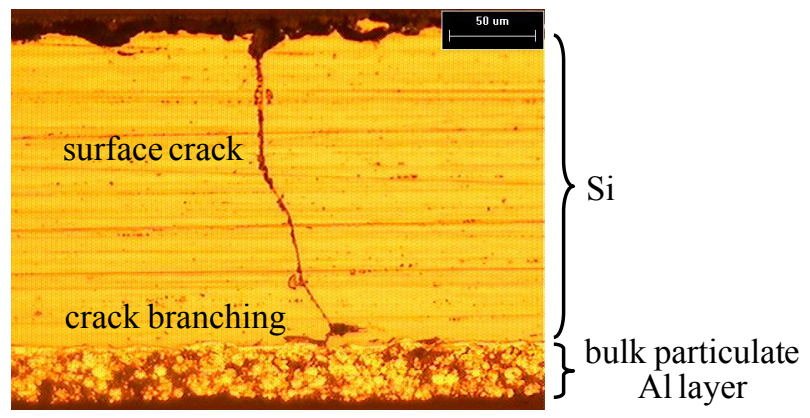

Figure 1. Optical micrograph of the cross section of a cracked silicon PV cell.

In the usual experimental arrangement for ESPI, two optical configurations are employed to measure the in-plane and out-of-plane displacement, respectively. It is found that bending displacements in the vicinity of cracks are larger than extensional deformations in such a thin plate ${ }^{[8]}$. This work focuses on the latter measurement. Heating cycles with small amplitude of fluctuation were applied to the edge-supported specimens to induce bending. Out-of-plane ESPI evolved from speckle interfermetry is based on the coherent addition of the scattered light from the object surface and a reference beam, which may be a specular or scattered field not necessarily originating from the object. The phase change caused by object displacement within one speckle is coded by the reference beam under the assumption of an altered surface. This displacement is extracted by correlating two speckle patterns, one taken before and the other taken after object displacement. The intensity recorded in the first frame, which is distributed at a point in the observation plane before the object is deformed.

Figure 2 depicts a schematic diagram of ESPI configuration for out-of-plane displacement measurement. This optical measurement system is similar to Michelson interferometer. A laser beam passing through a spatial filter and beam splitter is used to illuminate the specimen and the reference plane. The experimental light source was a DPSS laser st a wavelength of $532 \mathrm{~nm}$. The CCD camera is equipped with a Sony ICX625 image sensor which is highly sensitive to this wavelength. The optical beams reflected from the specimen and reference plane cross the beam splitter to produce an interference fringe pattern and were captured by a high-resolution CCD camera having $2448 \times 2050$ pixels. The CCD camera signal was recorded by a personal computer. A temperature controllable planar heater was used to apply heat flux to the object. The heater temperature was measured by J-type thermocouple through a NI-9213 module. The edgesupported specimen was heated to induce bending deflection. Specklegrams were taken at constant temperature 
increments till speckle fringes near the crack were sufficient to determine the crack length. All operation and detection devices were controlled by LabVIEW program.

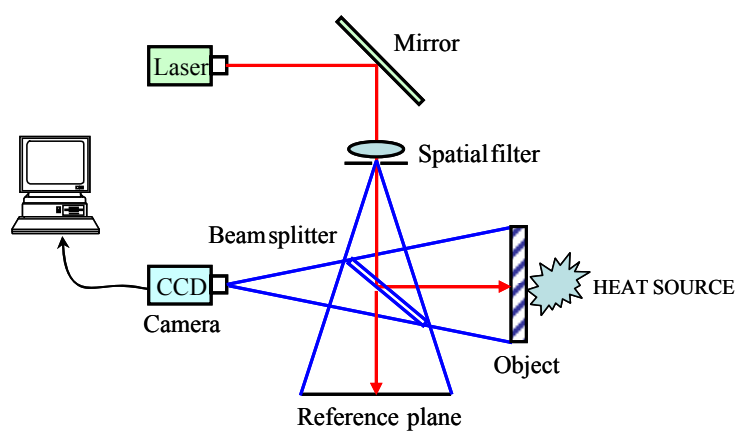

Figure 2. ESPI configuration for measuring out-of-plane displacement.

\section{RESULTS AND DISSCUSSION}

To seek the appropriate operating temperature range and increment for a prominent speckle pattern correlated to thermal deformation, one photograph was captured at every $0.2^{\circ} \mathrm{C}$ increment during the heating process applied on both undamaged and damaged crystalline silicon solar cells. The speckle patterns were determined by subtracting two pictures recorded at different temperatures and then performing image processing technique based on Fourier optics. Figure 3(a) shows the out-of-plane ESPI image of crystalline silicon solar cell without damage under thermal load. Numerous speckle fringes resulted from the initial out-of-plane bending deformation in the solar cell are due to different coefficients of thermal expansion in the silver strips and the silicon substrate. The fringe pattern was analyzed as a topographic map of the $\mathrm{z}$ coordinate on the specimen surface, where the contour interval is $\lambda / 2$ per fringe order, and $\lambda$ the illuminating source wavelength. The detection system has a sensitivity of $0.266 \mu \mathrm{m}$. The out-of-plane displacement was identified by FEA. The silicon and silver-strip were modeled by isoparametric solid elements. Figure 3(b) shows that the contours of out-of-plane displacement in the edge-supported PV cell due to $0.4^{\circ} \mathrm{C}$ temperature increment.

A feasibility study of ESPI inspection for various defects in PV cells was carried out numerically using a commercial FEA code ANSYS 13.0. The specimens considered in numerical simulation were poly-silicon cells with the dimension $156 \times 156 \times 0.2 \mathrm{~mm}$. The displacement field around a flaw is of greater interest than stress distribution near the crack tip in this study. We adopted the 8-node isoparametric solid elements SOLID45 in ANSYS instead of crack elements at the crack tip in computation. Three kinds of defects were considered. The scratch was simulated by removing the material

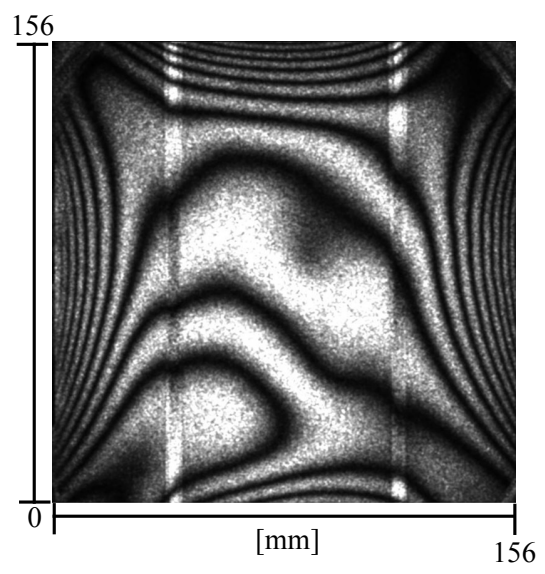

(a)

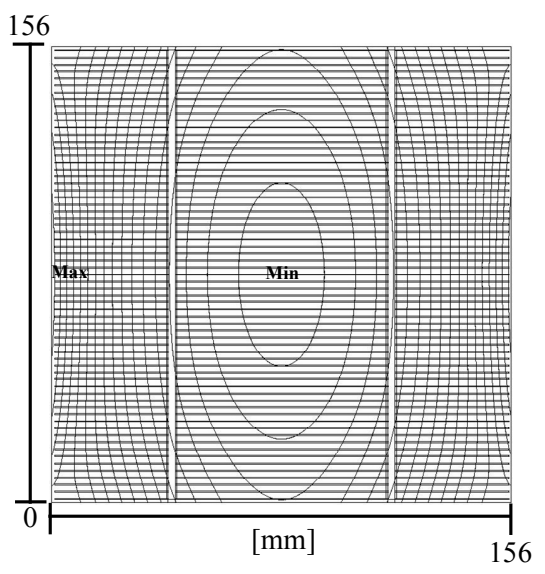

(b)

Figure 3. (a) Measured speckle fringes and (b) displacement contours calculated by FEA for out-of-plane deformation of a 156 mm square polycrystalline silicon solar cell induced by temperature rise from 32 to $32.4^{\circ} \mathrm{C}$. 
occupied a volume of $25 \mathrm{~mm}$ in length, $0.6 \mathrm{~mm}$ in width, and $0.01 \mathrm{~mm}$ in thickness from the cell surface. The surface crack was modeled as a line flaw having the depth less than cell thickness. Before the crack grows through the thickness, the cell surface remains continuous but its tangential discontinues across the crack. The through-thickness cracks are potentially dangerous defects in brittle materials such as silicon. Figure 4 shows a comparison of calculated out-of-plane displacement contours for the $25 \mathrm{~mm}$ long scratch, surface crack of $70 \%$ depth-to-thickness ratio, and through-thickness crack initiated at the middle of the right periphery in a poly-silicon solar cell. In this figure, the speckle patterns in the area close to the defects demonstrate that the fringes are dense, chevron shaped, and broken for scratches, surface cracks, and through-thickness cracks, respectively. The fringe patterns represent the contours of out-of-plane displacement in increments of $0.266 \mu \mathrm{m}$.

The influence of crack depth on fringe numbers for the temperature rises applied on the polycrystalline silicon solar cell is illustrated in Fig. 5(a). The number of calculated fringes over the crack length is nearly constant for the crack depth-tothickness ratio in the range below $70 \%$. Increasing temperature rise will cause an increase in the number of calculated fringes. As the depth of surface crack exceeds $70 \%$ of the cell thickness, the fringe number increases and the speckle fringes reveal the chevron shaped pattern across the crack. The intersection points indicate the free surface of the crack. The fringes are not segmented until the surface crack extends through the thickness. Figure 5(b) demonstrates that calculated fringe number induced by different temperature rises with respect to length of the surface crack extended from the middle periphery of the poly-silicon PV cell, in which the surface crack is of a half thickness depth. The number of fringes over the flawed area is proportional to both crack length and temperature rise applied on the cell. A moderate temperature rise induces dense fringes of fine spacing, which provide good accuracy for estimation of crack length.

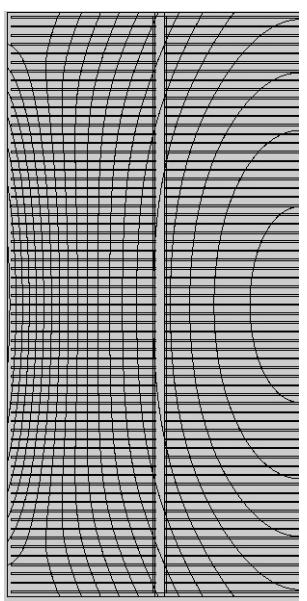

(a)

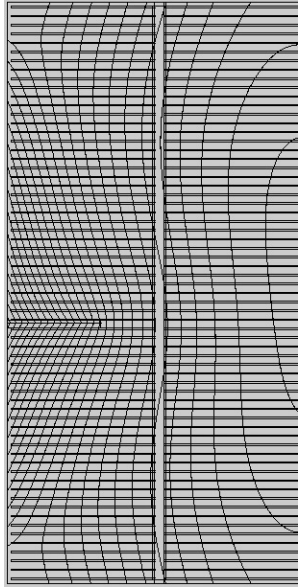

(b)

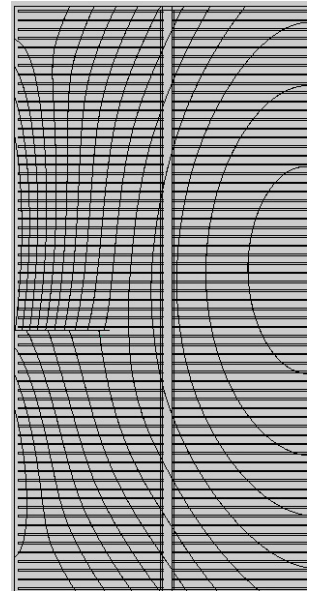

(c)

Figure 4. Zoomed images of calculated out-of-plane displacement contours for the $25 \mathrm{~mm}$ long (a) scratch, (b) surface crack of $70 \%$ depth-to-thickness ratio, and (c) through-thickness crack initiated at the middle of right edge in a poly-silicon solar cell with corresponding dimensions in Fig. 3.

(a)

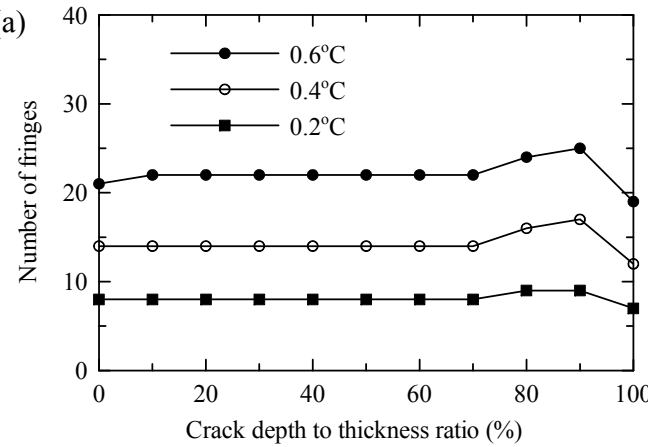

(b)

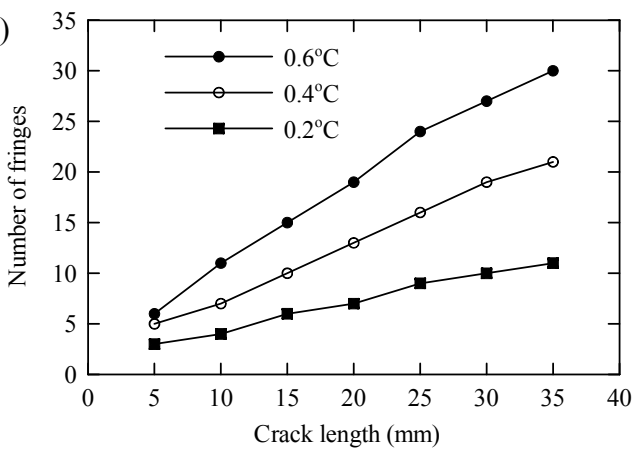

Figure 5. Influence of (a) crack depth and (b) temperature rise on calculated fringe numbers in a 156 mm square polycrystalline silicon solar cell with a $25 \mathrm{~mm}$ long surface edged crack extended from the middle periphery. 
Figure 6(a) shows the EL image of an edge-cracked poly-crystalline solar cell. A $38 \mathrm{~mm}$ long edge crack is visualized by a dark line in this photo. Figure 6(b) depicts the ESPI image of the same specimen shown in Fig. 6(a). The EL image is a mirror image of the ESPI image since the former was taken from the front face of PV cell. The back face of the cell is inspected by ESPI method since rough surface is rather suitable for ESPI. The defect position appears in the ESPI interferogram is identical to that in the EL image, but their orientations are revered. The accuracy of detection is unaffected by re-orientation. The speckle patterns shown in Fig. 6(b) substantially differ from those in Fig. 3(a). There clearly appear the chevron shaped fringes in the corresponding area occupied by the edge crack. The experimental results indicate that the edge crack did not grow through the thickness. The tangential slopes of the interference fringes are discontinuous near the crack. They are caused by discontinuous heat-induced displacement field across the crack surface. The crack length can be estimated in accordance with the region of discontinuity.

Figure 7(a) shows an EL image captured from the front face of a single-crystalline silicon solar cell, in which a $34 \mathrm{~mm}$ star crack was radiating from a common point inside the cell. The star crack was artificially created by the pyramidshaped diamond indenter. In an X-cut single-crystalline silicon wafer, the crack extension inherently takes place along the $\{110\}$ orientation. As expected, the displacement field in the vicinity of the crack is discontinuous. It results that the fringe pattern recorded from the back face appears distorted in a polygonal shape shown in Fig. 7(b). The diagonals connecting the opposing vertices indicate the braches of the star cracks.

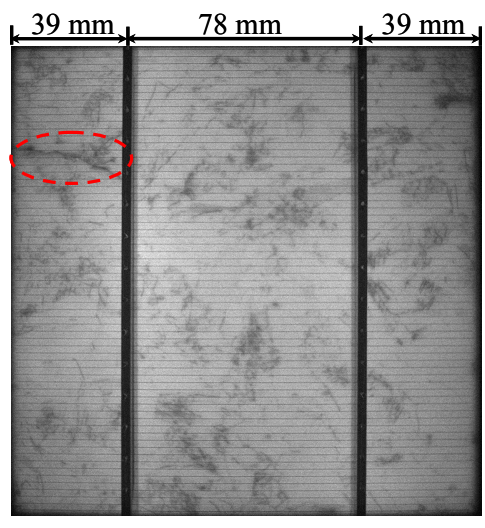

(a)

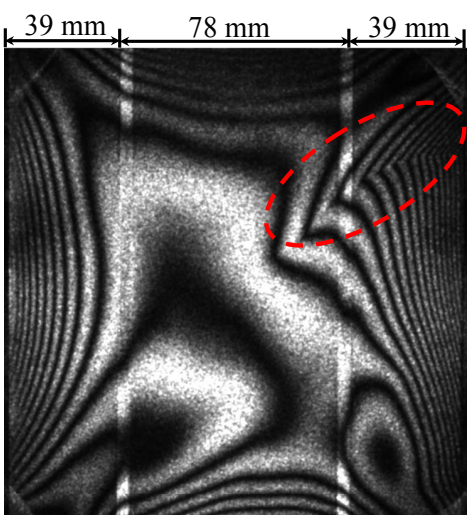

(b)

Figure 6. (a) EL image (front view) and (b) ESPI image (back view) of a $156 \mathrm{~mm}$ square poly-crystalline silicon solar cell with a 39 $\mathrm{mm}$ long edge crack. The visualization of a fringe pattern is produced by subtraction of two speckle patterns recorded at 32 and $32.4^{\circ} \mathrm{C}$.

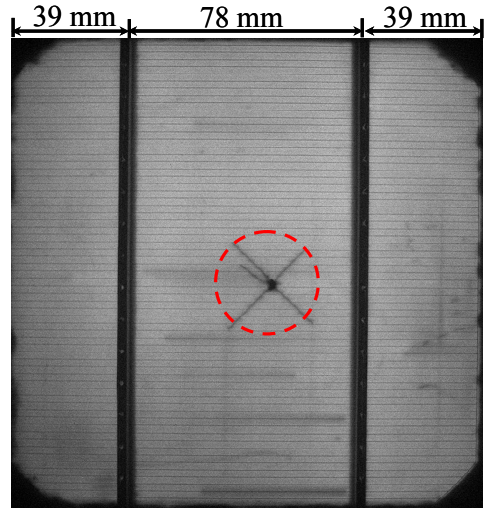

(a)

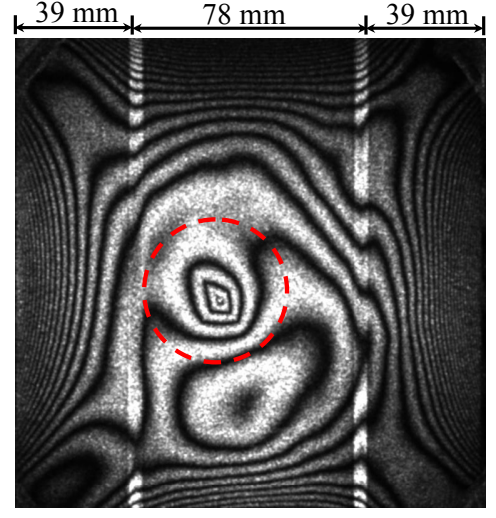

(b)

Figure 7. (a) EL image (front view) and (b) ESPI image (back view) of a $156 \mathrm{~mm}$ square single-crystalline silicon solar cell with a 34 $\mathrm{mm}$ long interior star crack. The fringe pattern is obtained by subtraction of two speckle patterns recorded at 32 and $32.4^{\circ} \mathrm{C}$. 


\section{CONCLUSION}

A rapid, full-field, nondestructive method of inspecting cracks in crystalline silicon PV cells was presented. This method is based on the ESPI technique and thermally induced flexural deflection of the specimen. Experimental and numerical results demonstrate that, under similar constraints and temperature rise, defect-free specimens have similar speckle patterns despite the inherent flexures in the cells. In flawed specimens, the interference fringes associated with heatinduced deflection clearly differ from those captured in undamaged specimens. The mechanical defect in a silicon PV cell includes scratch, surface crack, through-thickness crack, etc. A real crack could be branched or deflected in the thickness or the plane of PV cell, but it was simply modeled as a straight line defect in the numerical investigation. Numerical results indicate that broken speckle fringes and chevron shaped patterns are definitely observed in the near crack field for both through-thickness cracks and surface cracks of depth exceeding $70 \%$ of total thickness. The calculated speckle patterns have been validated by the ESPI measurements. The speckle patterns can characterize the crack length and location. In the present experimental system, cracks shorter than $10 \mathrm{~mm}$ were accurately detected, and the tolerance was $2 \mathrm{~mm}$.

In PV cells, surface scratches degrade electrical performance but do not substantially change mechanical displacement under the thermal load. One exclusive advantage of this method is the capability to distinguish between cracks and surface scratches. Crystalline silicon solar cells generally exhibit high thermal conductivity. The temperature increase required for inducing moderate speckle fringe density near a crack field is no more than 0.4 degree Celsius. Only simple configuration of heating process is needed to provide a fast temperature rise on the cells. This method is in progress coordinated with machine vision technology and might be the most possible technique for on-line PV cell sorting before or during module fabrication.

\section{ACKNOWLEDGMENT}

Financial support from Hsinchu Science Park Administration, National Science Council, Taiwan, was gratefully acknowledged.

\section{REFERENCES}

[1] Brun, X. F. and Melkote, S. N., "Analysis of stresses and breakage of crystalline silicon wafers during handling and transport," Sol. Energy Mater. Sol. Cells 93, 1238-1247 (2009).

[2] Würfel, P., Trupke, T., Puzzer, T., Schaffer, E., Warta, W. And Glunz, S. W., "Diffusion lengths of silicon solar cells from luminescence images," J. Appl. Phys. 101, 123110 (2007).

[3] Trupke, T., Bardos, R. A., Schubert, M. C. and Warta, W., "Photoluminescence imaging of silicon wafers," Appl. Phys. Lett. 89, 044107 (2006).

[4] Kasemann, M., Schubert, M. C., The, M., Köber, M., Hermle, M. and Warta, W., "Comparison of luminescence imaging and illuminated lock-in thermography on silicon solar cells," Appl. Phys. Lett. 89, 224102 (2006).

[5] Dallas, W., Polupan, O. and Ostapenko, S., "Resonance ultrasonic vibration for crack detection in photovoltaic silicon wafers," Semiconductor Science and Technology 21, 254-260 (2006).

[6] Hack, E. and Bronnimann, R., "Electronic speckle pattern interferometry deformation measurement on lightweight structure under thermal load," Optics and Lasers in Engineering 31, 213-222 (1999).

[7] Esteban, A. Z., Eden, C. G., Carlos, G. T. P., Ramon, R. V. and Hector, J. P. S., "Defect detection in metals using electronic speckle pattern interferometry," Sol. Energy Mater. Sol. Cells 88, 217-225 (2005).

[8] Zehnder, A. T. and Viz, M. J., "Fracture mechanics of thin plates cracked and shells under combined membrane, bending and twisting loading," Applied Mechanics Reviews 88, 37-48 (2005). 